\title{
A Quantitative Evaluation of Fixed-Pattern Noise Reduction Methods in Imaging Systems
}

\author{
Pablo Meza ${ }^{1,3}$, César San Martin ${ }^{2,3}$, Esteban Vera ${ }^{1,3}$, and Sergio Torres ${ }^{1,3}$ \\ 1 Depto. Ing. Eléctrica, Universidad de Concepción, Casilla 160-C, Concepción, Chile \\ 2 Depto. Ing. Eléctrica, Universidad de La Frontera, Casilla 54-D, Temuco, Chile \\ csmarti@ufro.cl \\ 3 Center for Optics and Photonics, Universidad de Concepción, Concepción, Chile
}

\begin{abstract}
Fixed-pattern noise is a common feature in several uncalibrated imaging systems, and it typically appears as striping and gridlike nonuniformity artifacts in hyperspectral and infrared cameras. In this work, we present a quantitative and comparative analysis of fixedpattern noise reduction, or calibrating techniques, by using several image quality indexes. A special emphasis is made in demonstrating the correspondence between the reference-free (blind) image quality indexes and the typical reference-based metrics, specially when using online calibration procedures where reference data is not available. We evaluate the performance of several classic scene-based calibrating algorithms applied to: multispectral images with simulated striping noise; and infrared image sequences with simulated nonuniformity. The results show that most of the tested reference-free indexes are useful indicators for tracking some of the real degradation of the calibrated or even uncalibrated imagery, but they are far from perfect to match an error or similarity measure if the clean or reference data is available.
\end{abstract}

\section{Introduction}

Digital imaging systems are often composed of a bundle of optical lenses and a focal-plane array (FPA) with its associated readout electronics. A FPA can be arranged in a one dimensional array, or linear detector, which might need to employ a scanning acquisition mode in order to form an image. On the other hand, a two dimensional FPA can directly form images without any moving parts, which is the case of most of the photographic and video cameras available on the market. However, in multi or hyperspectral cameras the two dimensional FPA is employed as an array of linear detectors, one for each wavelength band, so again there is a need for scanning in order to create the bidimensional images, which is the case from consumer RGB scanners up to state-of-the-art satellite cameras.

The fixed-pattern noise (FPN) corresponds to a degradation common to all FPA based imaging systems, and it is due to the intrinsic and uneven response of the individual detectors, or pixels, within the array. In the case of bidimensional staring arrays, such as in infrared cameras, the FPN is often spatially distributed

I. Bloch and R.M. Cesar, Jr. (Eds.): CIARP 2010, LNCS 6419, pp. 285-294, 2010.

(C) Springer-Verlag Berlin Heidelberg 2010 
in a grid-like appearance, known as nonuniformity (NU) noise. On the other hand, when dealing with a scanning device such as a hyperspectral imager, the FPN is seen as vertical or horizontal strip lines, known as striping noise, and the pattern is different between bands because each band is captured by a different line of the detector array.

Fortunately most of the detectors can be modeled by a linear model represented by a gain and an offset parameter. Therefore, the proper way of calibrating the FPA, and thus removing for the FPN, is by estimating such parameters and correct for their disparity or nonuniformity. Even though the FPN can be restored by a proper calibration of the detectors in order to estimate its intrinsic parameters, there are still two main issues. The first is related to the change on the environmental or operational conditions of the camera, so the parameters may drift. The second is related to the fact that the calibration may be unfeasible due to increased setup costs or complexity.

Nonetheless, several methods have been developed in order to calibrate the FPA and then reduce the striping and NU noise by using scene-based data or statistics. For the striping FPN, a moment matching (MM) method has been proposed in 3. In this case, the mean and variance of the readout data is obtained and used to compensate the corrupted image. In 9 a method based on histogram matching with weighted least-squares filter is introduce in order to reduce the striping noise in MODIS data. The histogram matching is used to reduce the detector-to-detector stripe and mirror stripes, and the weighted least-squares filter is used to reduce the stripe noise. In [1] several methods are reviewed and compared such as the ones based on: low pass filtering, gray value substitution, and wavelet transforms. In this case, the best visual results were obtained using a wavelet approach, but the use of a low pass filter had a noticeable noise removal in despite of removing useful high pass information. From this point, in [8] is proposed a combination of wavelet transform and frequency filtering in a novel, fast and stable filter called the wavelet-FFT (WFT) filter. For vertical striping noise, the vertical detail band contains the principal striping artifacts. Therefore the filter operation is only selectively applied to this band by means of a Gaussian low-pass filter, and the denoised image is then reconstructed using the inverse wavelet transform. The results show a good performance in visual results and radiometric range preservation. In order to reduce the FPN in staring FPA, such as in infrared imaging system, several methods have been proposed in the literature called nonuniformity correction (NUC) methods. In this work, we are interested in scene-based methods, which only make use of the images captured during the normal operation of the camera to perform the NUC, and thus removing the NU noise. Among all NUC methods, the following two are highlighted due to their simplicity and because they allow a dynamic frame-by-frame operation. The constant statistics algorithm [5], which has a reminiscence of the MM approach previously reviewed for scanning imaging systems, and the neural-network approach [12]13] which has a extreme ability for adapting to the drift of the parameters. 
Figures of merit for evaluating the performance of destriping or NUC methods can be divided in: reference-based and reference-free (or blind) performance metrics. Reference-based metrics require a set of reference images or video sequences. An example of this metric, which is used for laboratory calibration, is the correctability parameter [11. This parameter, defined as the ratio between the magnitudes of the spatial and temporal noise, indicates when the spatial noise has been reduced to a magnitude below the magnitude of the temporal noise. Another kind of reference-based metrics are the image quality indexes based on measure of distance, traditionally used in image processing such as the mean-squared error (MSE), the mean-absolute error (MAE), the root-mean-square-error (RMSE), and the peak-signal-to-noise-ratio (PSNR). The main advantage of such metrics is that they provide a radiometric performance evaluation of the algorithms, although globally. Lately, the universal image quality index (UIQI) 15, the structural similarity index measure (SSIM) 16] and the feature based SSIM [7] have been used in order to assess the quality of images in a perceptual framework. When the reference images are not available, the applicability of reference-based metrics is confined to simulation scenarios where artificial noise is added to sets of clean or previously calibrated images. On the other hand, a reference-free metric may be able to recognize quantitatively, but blindly, if a image is getting better or worst after any denoising or restoration procedure, trying to emulate our own visual system mechanism for deciding wether an image has a good overall quality or not. Among them, the most suited for evaluating the removal of FPN are the roughness $(\rho)$ index, the noise reduction (NR) ratio and the residual non-uniformity (RNU). The $\rho$ metric was introduced by Hayat's group in [6], and it quantifies the tentative amount of $\mathrm{NU}$ in an image by using first order gradient filters in the horizontal and/or vertical directions. The $\rho$ index is a clear indicator on the FPN removal, but it might produce confusion, leading to good (or low results) if real high frequency information from the original images is removed as well. A modified and enhanced version that accounts for some of the $\rho$ index flaws is also proposed in 10. The NR ratio was proposed and used in [2 in order to specifically evaluate the noise reduction achieved by the exemplified destriping method, and the index corresponds to the ratio between the mean frequency power of the raw image and the denoised one. Latest, the RNU index was defined in [14] to evaluate the ability of their own NUC method to reduce the FPN.

In this paper we use several image quality indices in order to compare and rank the performance of certain classic destriping and NUC methods using image data with simulated FPN. In Section 1 we present the striping noise problem and its reduction methods, and in Section 3 we explain the NU problem and the correction methods herein used. Section 4 contains a description of the quantitative indexes, and the results achieved by the simulations are summarized in Section 5. Finally, in Section 6 we present a discussion and some conclusions, as well as a outline of our future work. 


\section{Striping Noise and Its Reduction}

When the image is formed by the scanning of a one dimensional sensor array, the produced FPN is known as striping noise. Formally, by assuming a linear model between the readout signal $Y_{j}(n)$ and the input irradiance $X_{j}(n)$ collected by the $(j)^{\text {th }}$ detector at a given time $n$, it is common to define the following image formation model:

$$
Y_{j}(n)=A_{j}(n) \cdot X_{j}(n)+B_{j}(n),
$$

where $A_{j}(n)$ and $B_{j}(n)$ are the gain and the offset of the $j^{\text {th }}$ detector. As the gain and offset are not necessarily equal along the detector array, then any disparity presented between their responsivity and bias levels triggers the generation of a striping noise at the scanning direction. Hopefully, as the gain and offset are often constant in time, they can be computed by performing a calibration procedure, and further used for compensating the acquired images. However, it is well known that at different operation conditions, and specially at longer wavelengths, the gain and offset parameters may drift and thus differ from the previously calibrated ones. In addition, calibration setups can be expensive, as well as time consuming, and they are not always available, as it is the case of space-based applications such as in satellite imaging. Nevertheless, there are calibration methods that purely rely on the acquired data for estimating the uneven parameters, and thus reduce the undesired FPN.

Assuming the model en Eq. 1, it is possible to obtain the temporal mean and standard deviation of the readout data as:

$$
\begin{array}{r}
\mu_{Y_{n j}}=A_{j} \cdot \mu_{X_{n j}}+B_{j}, \\
\sigma_{Y_{n j}}=A_{j} \cdot \sigma_{X_{n j}},
\end{array}
$$

In addition, it is also feasible to assume that the input irradiance is of zero mean and unitary variance, obtaining that:

$$
\begin{aligned}
B_{j} & =\mu_{Y_{n j}}, \\
A_{j} & =\sigma_{Y_{n j}} .
\end{aligned}
$$

Thus, the image with reduced striping noise is finally given by:

$$
\hat{X}_{n j}=\left(Y_{n j}-\mu_{Y_{n j}}\right) / \sigma_{Y_{n j}} .
$$

This destriping noise reduction methods is known as the MM [3]. Now, if we apply the Fourier transform to the gain and offset images previously obtained we have that $F A(u, v)=\sum_{m=0}^{M-1} a_{m} e^{-j m v} \delta(u)$ and $F B(u, v)=\sum_{m=0}^{M-1} b_{m} e^{-j m v} \delta(u)$, representing that the gain and the offset is concentrated in the horizontal frequency components when vertical striping noise is present. Using this result, a WFT filter was proposed in [8], that applies a high pass filter to any vertical sub-band of a wavelet decomposition. For achieving good results, the WFT method relies on the adjustment of the two key parameters: the damping factor (of the high pass filter) and the decomposition level of the wavelet transform. In this paper, we use the best combination as reported in [8]. 


\section{Nonuniformity and Its Correction}

Assuming that each infrared detector is characterized by a linear model, the measured readout signal $Y_{i j}$ at a given time $n$ can be expressed as for the $(i j)^{\text {th }}$ detector in the form:

$$
Y_{i j}(n)=A_{i j}(n) \cdot X_{i j}(n)+B_{i j}(n),
$$

where $A_{i j}(n)$ and $B_{i j}(n)$ are the gain and the offset of the $i j^{\text {th }}$ detector, and $X_{i j}(n)$ is the real incident infrared photon flux collected by the respective detector.

Harris et. al in [4 developed a recursive version of the constant statistics NUC technique where the parameters are recursively estimated frame by frame as follows:

$$
\begin{aligned}
& B_{i j}(n+1)=\hat{\mu}_{Y_{i j}}(n+1)=\left(Y_{i j}(n+1)+n \hat{\mu}_{Y_{i j}}(n)\right) /(n+1) \\
& A_{i j}(n+1)=\hat{\sigma}_{Y_{i j}}(n+1)=\left(\left|Y_{i j}(n+1)-\hat{\mu}_{Y_{i j}}(n+1)\right|+n \hat{\sigma}_{Y_{i j}}(n)\right) /(n+1)
\end{aligned}
$$

A second fundamental NUC method is the one proposed by Scribner et. al in [12, where equation (7) is reordered as follows:

$$
X_{i j}(n)=w_{i j}(n) \cdot Y_{i j}(n)+b_{i j}(n),
$$

where the new parameters $w_{i j}(n)$ and $b_{i j}(n)$ are related to the real gain and offset parameters of each detector as follows:

$$
w_{i j}(n)=g_{i j}^{-1}(n) \quad b_{i j}(n)=-o_{i j}(n) g_{i j}^{-1}(n) .
$$

The expression presented in equation (8) is the responsible of performing the NUC on the readout data. Then, for each $i j^{\text {th }}$ detector, the NUC model (8) can be considered as the simplest neural network structure, which consists of a single linear neuron node, with an estimate weight $\left(\hat{w}_{i j}(n)\right)$ and an estimate bias $\left(\hat{b}_{i j}(n)\right)$. For this reason we denoted this method as neural network (NN). A complete version of Scribner's NUC method can be found in [13.

\section{Quality Indexes}

In this section, the quality indexes are addressed. The RMSE reference-based index for a $L$-dimensional FPA is given by:

$$
R M S E^{2}=\frac{1}{\operatorname{size}(L)} \sum_{L}\left(I_{L}-\hat{X}_{L}\right)^{2}
$$

where $\hat{X}$ is the corrected image and $I$ is the real image. $L$ is the dimension of the FPA array ( 1 or 2 ) where $I$ denote a vector or matrix, respectively. The RMSE is higher when the correction is poor, and it is lower when the corrected image is closer to the real image. 
Moving to reference-free indexes, the $\rho$ index is described as:

$$
\rho(\hat{X})=\left(\|h \otimes \hat{X}\|+\left\|h^{T} \otimes \hat{X}\right\|\right) /\|\hat{X}\|,
$$

where $\hat{X}$ is an $M \times N$ pixels compensated image, $\|\hat{X}\|$ is the $\ell_{1}$ norm of $\hat{X}, \otimes$ represents the discrete $2 \mathrm{D}$ convolution, $h$ is a horizontal edge-detector filter, which is defined as $[-11]$, and $T$ stands for vector transposition. Note that the roughness index measures the horizontal and vertical components of the FPN. If the stripe noise is presented in vertical direction, the $\rho$ index only requires the horizontal component. By definition, the roughness index is always a non-negative real number, and $\rho(\hat{X})=0$ whenever $\hat{X}$ is constantly flat. Thus, according to the roughness index, the closer to zero the better the NUC performed on the raw image, but if it reaches a zero value, it means that the corrected image is a constant and uninformative image.

In [10] the effective roughness (ER) index is proposed as a redefinition of the roughness index, modifying the edge-detection filtering operation. The mathematical definition of the ER index is:

$$
\operatorname{ER}(\hat{X})=\frac{\|h \otimes(g \otimes \hat{X})\|+\left\|h^{T} \otimes(g \otimes \hat{X})\right\|-2\|g \otimes \hat{X}\|}{\|g \otimes \hat{X}\|},
$$

where $g$ is any edge-detector filter. The rationale for the filtering operation is twofold: diminish the low-frequency components of the image and accentuate the (residual) noise in the image. The ER index ranks a correction with the best performance when $\operatorname{ER}(\hat{X})=0$.

Another method is the ratio of NR that has been used in several works [2]. This is defined as:

$$
N R=N_{0} / N_{1},
$$

where $N_{0}$ and $N_{1}$ stand for the mean power spectrum of the corrupted and corrected image, respectively. In order to quantify the performance of the denoising method, $N R \rightarrow \infty$, i.e., the ideal is to remove as much noise as possible from the corrupted image. Finally, the RNU metric is given by:

$$
R N U=\frac{1}{\mu}\left(\frac{1}{n m} \sum_{i} \sum_{j}\left(Y_{i j}-\mu\right)\right)^{1 / 2} \times 100 \%
$$

where $\mu$ is the spatial mean of the corrected image. The RNU must be lower in order to rank the best correction method.

Table 1. Statistical parameters to generate the simulated fixed-pattern noise test images and image sequences for both types of fixed pattern noise

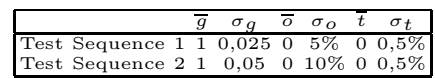




\section{Results}

In order to quantify the image quality indexes behavior, we present results over simulated FPN of both striping and NU, providing the real image and the corrupted frame. We compute the RMSE, $\rho$, ER, NR, and RNU metrics for the original images, and for two FPN reduction algorithms considered for both simulations. The destriping is performed using MM and WFT, and the NUC is performed by the constant statistics (CS) and NN methods. Two levels of FPN were chosen to generate the simulated fixed gain and offset image masks to produce the desired FPN appearance over each kind of data, and the selected statistics are the same for both types of noise, as displayed in Table. 11 In addition, a small temporal noise component was added to simulate the common electronic/thermal noise.

For the stripping noise simulation we used a multispectral satellite image with three channels (red, green and blue), using a different strip noise pattern with the same statistics to each channel. The main reason for this is to demonstrate how the correction affects the combined image, making easy to visualize any radiometric problems in the denoised images. For the infrared NU noise, we used a sequence of 4000 frames that includes camera motion and moving targets, so helping to achieve the requirements needed for the NUC algorithms. Fig. 1 1 shows graphically the FPN generated for both kinds of noise.

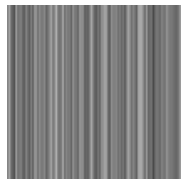

(a)

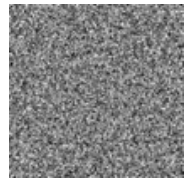

(b)

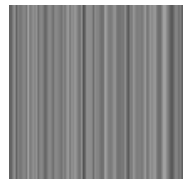

(c)

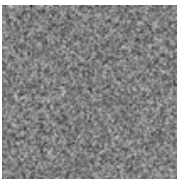

(d)

Fig. 1. Image of the simulated fixed pattern noise with $\bar{g}=0.025$ and $\sigma_{g}=5 \%$ : a) unidimensional and b) bidimensional gain; c) unidimensional and d) bidimensional offset

Table 2. Performance comparison of the striping reduction methods in terms of the different quality indexes

\begin{tabular}{|c|c|c|c|c|c|c|c|c|c|}
\hline \multicolumn{2}{|c|}{ Gain } & \multicolumn{7}{|c|}{$\sigma=5 \%$} & \multicolumn{3}{c|}{$\sigma=10 \%$} \\
\hline \multicolumn{2}{|c|}{ Offset } & \multicolumn{3}{|c|}{$\sigma=5 \%$} & \multicolumn{3}{c|}{$\sigma$} \\
\hline Method & Index & Ch1 & Ch2 & Ch3 & Mean & Ch1 & Ch2 & Ch3 & Mean \\
\hline Noisy & RMSE & 0.05 & 0.05 & 0.05 & $\mathbf{0 . 0 5}$ & 0.10 & 0.10 & 0.10 & $\mathbf{0 . 1 0}$ \\
& $\rho$ & 0.35 & 0.27 & 0.33 & $\mathbf{0 . 3 2}$ & 0.48 & 0.42 & 0.50 & $\mathbf{0 . 4 7}$ \\
& ER & 0.54 & 0.58 & 0.59 & $\mathbf{0 . 5 7}$ & 0.67 & 0.73 & 0.71 & $\mathbf{0 . 7 0}$ \\
& NR & 1.00 & 1.00 & 1.00 & $\mathbf{1 . 0 0}$ & 1.00 & 1.00 & 1.00 & $\mathbf{1 . 0 0}$ \\
& RNU & 0.81 & 0.66 & 0.85 & $\mathbf{0 . 7 7}$ & 0.86 & 0.72 & 0.89 & $\mathbf{0 . 8 2}$ \\
\hline MM & RMSE & 0.06 & 0.06 & 0.07 & $\mathbf{0 . 0 6}$ & 0.06 & 0.07 & 0.08 & $\mathbf{0 . 0 7}$ \\
& $\rho$ & 0.27 & 0.20 & 0.28 & $\mathbf{0 . 2 5}$ & 0.29 & 0.22 & 0.29 & $\mathbf{0 . 2 7}$ \\
& ER & 0.32 & 0.37 & 0.39 & $\mathbf{0 . 3 6}$ & 0.33 & 0.37 & 0.39 & $\mathbf{0 . 3 6}$ \\
& NR & 0.94 & 0.93 & 0.86 & $\mathbf{0 . 9 1}$ & 0.88 & 0.85 & 0.80 & $\mathbf{0 . 8 4}$ \\
& RNU & 0.81 & 0.66 & 0.85 & $\mathbf{0 . 7 7}$ & 0.86 & 0.72 & 0.89 & $\mathbf{0 . 8 2}$ \\
\hline WFT & RMSE & 0.02 & 0.02 & 0.02 & $\mathbf{0 . 0 2}$ & 0.02 & 0.02 & 0.02 & $\mathbf{0 . 0 2}$ \\
& $\rho$ & 0.25 & 0.19 & 0.23 & $\mathbf{0 . 2 2}$ & 0.26 & 0.19 & 0.22 & $\mathbf{0 . 2 2}$ \\
& ER & 0.33 & 0.37 & 0.38 & $\mathbf{0 . 3 6}$ & 0.33 & 0.37 & 0.38 & $\mathbf{0 . 3 6}$ \\
& NR & 1.00 & 1.00 & 1.00 & $\mathbf{1 . 0 0}$ & 1.00 & 1.00 & 1.00 & $\mathbf{1 . 0 0}$ \\
& RNU & 0.78 & 0.64 & 0.82 & $\mathbf{0 . 7 5}$ & 0.78 & 0.64 & 0.79 & $\mathbf{0 . 7 4}$ \\
\hline
\end{tabular}


Results obtained for the two destriping corrections used for each channel, and for different levels of FPN, are summarized in Table. 2, where the mean value obtained from the three channels is calculated to compare the methods. The RMSE analysis shows that the WFT correction produces a more similar image compared to the original one, and that the increase of the FPN affects in the same amount both corrections. When comparing the reference free indexes, $\rho$ and ER fail to difference which correction generates a better result without indicating any change after applying the higher FPN mask. On the other hand, RNU presents mixed results, correctly interpreting the FPN reduction using the WFT technique, but does not reveals the increase in the FPN applied. The NR values cannot be correlated with the other results because it indicates that the destriping effects of the algorithms does not produce any different output whatsoever. It is necessary to highlight that in the test sequence 1 the MMcorrection produce a worse image than the noisy one product of the change in the radiometric range but all the reference free indexes fail to indicate. Fig. 2 presents the noisy image and the FPNcorrected versions.

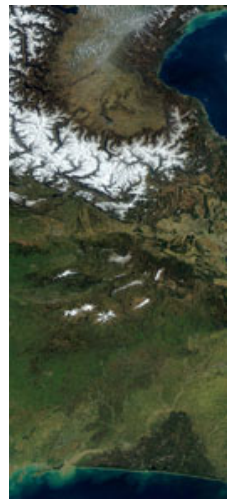

(a)

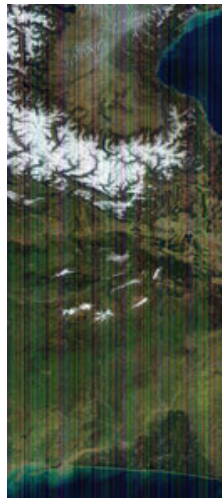

(b)

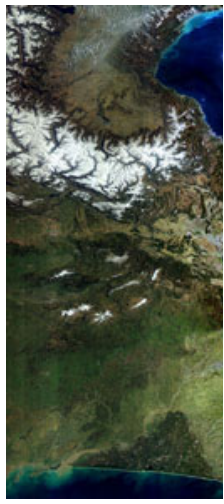

(c)

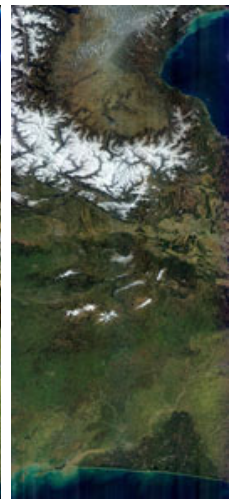

(d)

Fig. 2. Destriping results on simulated FPN (test sec. 2) in the reconstructed color image a) Reference Image b) Noisy Image c) MM-Correction and d) WFT-Correction

For the infrared case, the mean and standard deviation of every index calculated over 2000 frames after the algorithms have reached the stationary state are presented in Table. 3|(a), and a sample for the frame 1602 is shown in Table.(b). The main observation for the indexes values is that they follow a similar behavior between the results reported for the bidimensional FPN case as in the one dimensional FPN case. The results in Table. 3[(b) show a scenery where the indexes indicate incongruous results, where Harris NUC method produces a corrected image that is worse than the original, but the $\rho$ and ER index indicate a minor effect of the FPN than the original. At the same time, the NR and RNU index are not delivering new information when the level of FPN is increased. Fig. 3 presents the noisy corrected frame 1602 of the infrared video sequence. 
Table 3. a) Mean/standar deviation and b) Frame 1602 comparison performance of the non-uniformity reduction methods in terms of the different quality indexes

(a)

\begin{tabular}{|c|c|c|c|}
\hline \multicolumn{2}{|c|}{ Gain } & \multicolumn{2}{c|}{$\sigma=0.02$} \\
\hline \multicolumn{2}{|c|}{ Offset } & $\sigma=5 \%$ & $\sigma=10 \%$ \\
\hline Method & Index & mean/std & mean/std \\
\hline Noisy & RMSE & $0.05 / 0$ & $0.10 / 0$ \\
& $\rho$ & $0.35 / 0.05$ & $0.62 / 0.12$ \\
& ER & $0.82 / 0.07$ & $0.94 / 0.37$ \\
& NR & $1.00 / 0.00$ & $1.00 / 0.00$ \\
& RNU & $0.37 / 0.04$ & $0.43 / 0.05$ \\
\hline Harris & RMSE & $0.12 / 0.04$ & $0.12 / 0.04$ \\
& $\rho$ & $0.20 / 0.10$ & $0.20 / 0.11$ \\
& ER & $0.22 / 0.05$ & $0.23 / 0.06$ \\
& NR & $1.13 / 0.09$ & $1.36 / 0.18$ \\
& RNU & $0.21 / 0.07$ & $0.49 / 0.14$ \\
\hline NN & RMSE & $0.01 / 0.002$ & $0.02 / 0.004$ \\
& $\rho$ & $0.13 / 0.02$ & $0.14 / 0.02$ \\
& ER & $0.21 / 0.05$ & $0.27 / 0.06$ \\
& NR & $1.12 / 0.04$ & $1.36 / 0.11$ \\
& RNU & $0.35 / 0.04$ & $0.35 / 0.04$ \\
\hline
\end{tabular}

(b)

\begin{tabular}{|c|c|c|c|}
\hline \multicolumn{2}{|c|}{ Gain } & \multicolumn{2}{c|}{$\sigma=0.02$} \\
\hline \multicolumn{2}{|c|}{ Offset } & $\sigma=5 \%$ & $\sigma=10 \%$ \\
\hline Method & Index & & \\
\hline Noisy & RMSE & 0.05 & 0.10 \\
& $\rho$ & 0.28 & 0.48 \\
& ER & 0.8 & 0.94 \\
& NR & 1.00 & 1.00 \\
& RNU & 0.42 & 0.45 \\
\hline Harris & RMSE & 0.11 & 0.11 \\
& $\rho$ & 0.13 & 0.14 \\
& ER & 0.28 & 0.31 \\
& NR & 1.01 & 1.16 \\
& RNU & 0.35 & 0.35 \\
\hline NN & RMSE & 0.02 & 0.03 \\
& $\rho$ & 0.12 & 0.14 \\
& ER & 0.25 & 0.32 \\
& NR & 1.12 & 1.29 \\
& RNU & 0.40 & 0.39 \\
\hline
\end{tabular}

The obtained results suggest that from all of the reference free metrics, the one that best track the evolution of the correction or denoising process is the $\rho$ index, in despite of the fact that it fails when identifying low frequency noise components or radiometric changes in the resulting image. In addition, it is the one that better fits the tendency given by the RMSE.

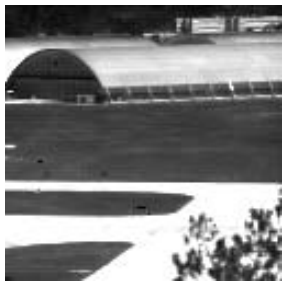

(a)

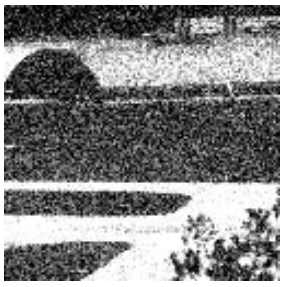

(b)

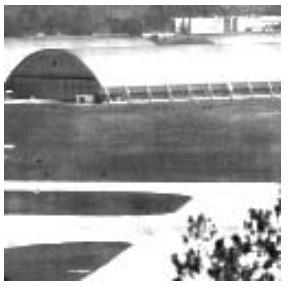

(c)

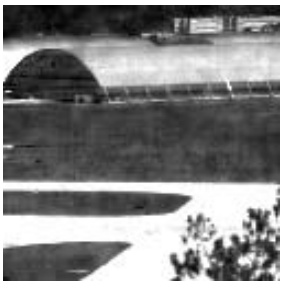

(d)

Fig. 3. NUC results on simulated FPN (test sec. 2) in the frame 1602 a) Reference Image b) Noisy Image c) Harris Correction and d) NN-Correction

\section{Conclusions}

In this paper we contrast several state-of-the-art reference-free indexes which are commonly used to evaluate the effectiveness of the FPN reduction methods. The study was performed by simulating the FPN typically found in two different imaging systems, such as hyperspectral and infrared cameras. The results indicate a low level of confidence between the presented indices given the inconsistency found when they are compared to each other. Nevertheless, the $\rho$ metric presents the best results as a good blind indicator when it is contrasted to a standard reference-based index, but unfortunately it is not able to reveal any modification to the radiometric or dynamic range of the corrected or calibrated images. Future work may include a deeper study for different types of 
indexes and FPN reduction methods, giving the foundations for the design of a new blind index for NUC purposes.

\section{Acknowledgements}

This work was partially supported by FONDECYT 11090034 and the Center for Optics and Photonics (CEFOP) FB0824/2008.

\section{References}

1. Chang-yan, C., Ji-xian, Z., Zheng-jun, L.: Study on Methods of Noise Reduction in a Stripped Image. Int. Arch. of P, RS, and SI Sciences 37, 213-216 (2008)

2. Chen, J., Shao, Y., Guo, H., Wang, W., Zhu, B.: Destriping CMODIS Data by Power Filtering. IEEE Trans. on Geoscience and Remote Sensing 41, 2119-2124 (2003)

3. Gadallah, F., Csillag, F.: Destriping Multisensor Imagery with Moment Matching. International Journal of Remote Sensing 21, 2505-2511 (2000)

4. Harris, J., Chiang, Y.: Nonuniformity correction using constant average statistics constraint: analog and digital implementation. In: Proc. of SPIE, vol. 3061, pp. 895-905 (1997)

5. Harris, J., Chiang, Y.: Nonuniformity correction of infrared image sequences using constant statistics constraint. IEEE Trans. on Image Proc. 8, 1148-1151 (1999)

6. Hayat, M., Torres, S., Amstrong, E., Cain, S., Yasuda, B.: Statistical algorithm for nonuniformity correction in focal plane arrays. Applied Optics 38, 773-780 (1999)

7. Liu, Z., Forsyth, D., Laganière, R.: A feature-based metric for the quantitative evaluation of pixel-level image fusion. C. Vision and Image Under. 109, 56-68 (2008)

8. Munch, B., Trtik, P., Marone, F., Stampanoni, F.: Stripe and ring artifact removal with combined wavelet Fourier filtering. Optics Express 17, 8567-8591 (2009)

9. Rakwatin, P., Takeuchi, W., Yasuoka, Y.: Stripe Noise Reduction in MODIS Data by Combining Histogram Matching With Facet Filter. IEEE Trans. on Geoscience and Remote Science 45(6), 1844-1856 (2007)

10. San Martn, C., Torres, S., Pezoa, J.: An Effective Reference-Free Performance Metric for Non-uniformity Correction Algorithms in Infrared Imaging Systems. In: Proc. of the IEEE LEOS 20th A. M., pp. 576-577 (2007)

11. Schulz, M., Caldwell, L.: Nonuniformity Correction and Correctability of infrared focal plane arrays. Infrared Phys. Technology 36, 763-777 (1995)

12. Scribner, D., Sarkady, K., Kruer, M.: Adaptive nonuniformity correction for infrared focal plane arrays using neural networks. In: Proc. of SPIE, vol. 1541, pp. 100-109 (1991)

13. Scribner, D., Sarkady, K., Kruer, M.: Adaptive retina-like preprocessing for imaging detector arrays. In: Proc. of the IEEE Int. Conf. on Neural Net., vol. 3, pp. 19551960 (1993)

14. Wang, B., Liu, S., Bai, L.: An enhanced non-uniformity correction algorithm for IRFPA based on neural network. Optics Communications 281, 2040-2045 (2008)

15. Wang, Z., Bovik, A.: A universal image quality index. IEEE SP L 9, 81-84 (2002)

16. Wang, Z., Bovik, A., Sheikh, H., Simoncelli, E.: Image quality assessment: from error visibility to structural similarity. IEEE T. on Image Proc. 13, 600-612 (2004) 